\title{
Coronavirus disease 2019: favorable outcome in an immunosuppressed patient with multiple sclerosis
}

\author{
Jens Devogelaere ${ }^{1,2}$ (D) $\cdot$ Marie B. D'hooghe ${ }^{1,2} \cdot$ Famke Vanderhauwaert $^{1} \cdot$ Miguel D'haeseleer $^{1,2}$
}

Received: 29 May 2020 / Accepted: 16 June 2020 / Published online: 20 June 2020

(C) Fondazione Società Italiana di Neurologia 2020

\begin{abstract}
The rapid and global spread of severe acute respiratory syndrome coronavirus 2, a viral pathogen responsible for the development of the "coronavirus disease of 2019" (COVID-19), has developed into an unprecedented health crisis with considerable case fatality rate. Patients with comorbidities are considered to be at higher risk for severe disease with acute respiratory failure, intensive care unit admission, and/or death. Particular vigilance has been warranted regarding the continuation of immunosuppressive treatments since viral clearing may be hampered in such cases. In contrast, it has also been hypothesized that overactive immune responses may trigger a cytokine storm associated with clinical deterioration, which has generated an interest in certain immunosuppressant drugs as potential treatment for COVID-19. We would like to present the first case report of a patient who was formally diagnosed with COVID-19 while being under disease-modifying treatment with rituximab, an anti-CD20 B cell depleting agent, for multiple sclerosis. The clinical picture was mild for which we have tried to provide an immunopathological framework.
\end{abstract}

Keywords COVID-19 $\cdot$ Immunosuppression $\cdot$ Multiple Sclerosis $\cdot$ Rituximab

\section{Case presentation}

A 33-year-old female patient with relapsing-onset multiple sclerosis (MS) was electively admitted to the Belgian Nationaal Multiple Sclerose Centrum for neurological assessment and multidisciplinary rehabilitation on March 9, 2020. Such programs are covered by the local reimbursement system and considered to be part of the standard medical care.

Jens Devogelaere

Jens.killian.devogelaere@vub.be; https://orcid.org/0000-00029410-581X

Marie B. D'hooghe

marie.dhooghe@mscenter.be

Famke Vanderhauwaert

famke.vanderhauwaert@mscenter.be

Miguel D'haeseleer

miguel.haeseleer@mscenter.be

1 Department of Neurology, Nationaal Multiple Sclerose Center, Vanheylenstraat 16, 1820 Melsbroek, Belgium

2 Department of Neurology; Center for Neurosciences, Vrije Universtiteit Brussel, Brussels 1090, Belgium
She was diagnosed with MS in 2004 following an episode of optic neuritis of her right eye. Subsequent relapses resulted in spastic paraparesis and cerebellar dysfunction presenting with truncal and appendicular ataxia. Off-label disease-modifying MS therapy with rituximab, an anti-CD20-mediated B cell depleting agent [1], was given as a single intravenous course of $1000 \mathrm{mg}$ on October 11, 2019, after a period of disease progression with clinical and radiological inflammatory activity, despite treatment with subcutaneous interferon-beta-1a. Expanded Disability Status Scale score was 8.0 at that time [2], and remained stable ever since. Seven days after admission, our patient developed fever, muscle soreness, headache, productive cough, and mild dyspnea. Blood analysis revealed an elevated C-reactive protein level $(35.6 \mathrm{mg} / \mathrm{L}$, normal value $<5.0 \mathrm{mg} / \mathrm{L})$, lymphopenia $\left(0.3 \times 10^{9} / \mathrm{L}\right.$, normal range 1.2 $\left.3.6 \times 10^{9} / \mathrm{L}\right)$, and neutropenia $\left(1.6 \times 10^{9} / \mathrm{L}\right.$, normal range $2.5-7.8 \times 10^{9} / \mathrm{L}$ ), whereas corresponding baseline values (routinely measured on the day of admission) all had been normal. Considering the recent "coronavirus disease of 2019" (COVID-19) outbreak [3], and after identifying a potentially contaminating visit, a nasopharyngeal swab for real-time reverse transcriptase polymerase chain reaction detection of severe acute respiratory syndrome coronavirus 2 (SARS-CoV2)-i.e., the culprit agent in COVID-19-was ordered and 
came back positive. Strict hygienic and in-hospital isolation measures were applied to reduce the risk of disease spread. The patient fully recovered under symptomatic management over approximately 1 week; blood oxygen saturation levels remained $>96 \%$ during the entire disease period. Blood sampling around that time demonstrated a (nearly) complete restoration of the abovementioned abnormalities. Additional cellular immunology, performed 1 week after the positive COVID-19 test, showed unaffected numbers of CD4+ and CD8+ T lymphocytes, as well as natural killer cells. CD19+ $\mathrm{B}$ cell levels were, as expected with rituximab, reduced to $0.3 \%$ of the total lymphocyte population (normal range 5.0 $20.0 \%$ ). Hypogammaglobulinemia in the $\mathrm{IgG}$ subfraction was observed $(7.11 \mathrm{~g} / \mathrm{L}$, normal range $7.51-15.60 \mathrm{~g} / \mathrm{L})$ and $\mathrm{D}-$ dimers were elevated $(738 \mu \mathrm{g} / \mathrm{L}$, normal value $<500 \mu \mathrm{g} / \mathrm{L})$, in accordance with previous data in COVID-19 patients [4].

\section{Discussion}

We present the first report of a patient with MS who was formally diagnosed with COVID-19 and experienced a favorable clinical outcome despite immunosuppressive therapy with rituximab. SARS-CoV-2 is a highly virulent pathogen with a considerable case fatality rate notwithstanding extensive global effort. Presumed risk factors for severe disease with development of acute respiratory distress syndrome include diabetes mellitus, arterial hypertension, heart failure, and chronic pulmonary disease [3]. Upon entering the body, the coronavirus rapidly infects alveolar epithelial cells through the angiotensin-converting enzyme 2 receptor. Toll-like, retinoic acid-inducible gene-I-like and pathogen-recognition receptors play a key role in initiating viral clearing by activation of downstream elements such as nuclear factor-kappa B, interferons, and interleukins [5]. These innate immune mechanisms are not expected to be deeply affected by rituximab, given its specific mode of action, but may become easily overturned by SARS-CoV-2, as, for instance, the N-protein located at the genomic level is a powerful antagonist of the host interferon system [5]. CD8+ T cell counts can rise up to $80 \%$ of total inflammatory cell population in the pulmonary interstitium during COVID-19, emphasizing the importance of this cell type in host defense [6]. Corresponding values were normal in our patient, likely contributing to an effective adaptive immune response. Humoral factors, mediated by the activation and maturation of naïve B-lymphocytes, are critically important to establish long-lasting immune recognition, but could be less significant in the initial antiviral response, translating to a good clinical recovery despite medicationinduced B cell lymphopenia. In another study, mice were infected with the related Middle East respiratory syndrome coronavirus, and a model was created with animals deficient in $\mathrm{T}$ cells, B cells or both. T lymphocyte deficiency was associated with absence of viral clearance, whereas mice lacking B cells were able to overcome the infection [7]. Theoretically, it might be hypothesized that some forms of immunosuppression offer a protective effect versus the cytokine release storm observed in COVID-19. Hence, certain cytokines (e.g., interleukin 2 and 7, granulocyte-colony stimulating factor, and tumor necrosis factor alpha) are upregulated during infection and are associated with adverse outcomes (i.e., need for intensive care unit admission) in affected subjects [8]. Modulating these pathways might prove beneficial in the approach of this disorder, yet, the precise role of rituximab in that regard remains to be elucidated.

Invasive viral disease has not emerged as a major concern from clinical trial development and postmarketing surveillance of anti-CD20 therapies in individuals with MS. Nonetheless, many national guidelines are currently recommending suspended dosing, where possible, because of the COVID-19 pandemic. Maximizing risk avoidance during a global infectious threat seems reasonable but we must remain equally vigilant for the return of disease activity in highly active MS. Our findings illustrate that SARS-CoV-2 infection does not necessarily translate into a poor prognosis in MS patients who are receiving B cell depleting agents, and are in line with recent reports of mild COVID-19 in subjects treated with ocrelizumab [9]. Additional data and more extensive evaluations, however, are required to make a more firm safety claim and, for a number of reasons, caution is warranted before generalizing our observations. First, rituximab and ocrelizumab are occasionally associated with severe neutropenia and/or more profound hypogammaglobulinemia [1], which may impose an additional stress on the immune system. Second, our patient had none of the other medical risk factors for worse COVID-19 prognosis (see above) and was a non-smoker. Third, B cell levels in her blood were no longer fully depleted $(0.3 \%)$ at the time of infection, as the previous rituximab administration dated from more than 4 months before. Fourth and finally, a uniform dosing scheme exists for ocrelizumab but this is less the case for rituximab, where, for example, 6-monthly intravenous courses of $2000 \mathrm{mg}$ (usually divided over two administrations of $1000 \mathrm{mg}$ each, with a 2-week interval) are not uncommon. At this moment, within the context of the ongoing COVID-19 crisis, we advocate for carefully balanced case-bycase treatment decisions, including monitoring of disease activity and CD19+ B cell repopulation, regarding anti-CD20 agents in subjects with MS.

\section{Compliance with ethical standards}

Conflict of interest Jens Devogelaere reports no disclosures. Marie B. D'hooghe received consultancy fees and travel support from Roche®. Famke Vanderhauwaert reports no disclosures. Miguel D'haeseleer received consultancy fees and travel support from Roche®. 
Ethical approval None

\section{References}

1. Ineichen BV, Moridi T, Granberg T, Piehl F (2020) Rituximab treatment for multiple sclerosis. Mult Scler 26(2):137-152

2. Kurtzke J (1983) Rating neurologic impairment in multiple sclerosis: an expanded disability status scale (EDSS). Neurology 33(11):1444 1452

3. Rothan HA, Byrareddy SN (2020) The epidemiology and pathogenesis of coronavirus disease (COVID-19) outbreak. J Autoimmun 109:102433

4. Gao Y, Li T, Han M et al (2020) Diagnostic utility of clinical laboratory data determinations for patients with the severe COVID-19. J Med Virol 92(7):791-796

5. Li G, Fan Y, Lai Y, Han T, Li Z, Zhou P, Pan P, Wang W, Hu D, Liu $\mathrm{X}$, Zhang Q, Wu J (2020) Coronavirus infections and immune responses. J Med Virol 92:424-432
6. Maloir Q, Ghysen K, von Frenckell C, Louis R, Guiot J (2018) Acute respiratory distress revealing antisynthetase syndrome. Rev Med Liege 73(7-8):370-375

7. Jincun Z, Kun L, Christine WL et al (2014) Rapid generation of a mouse model for Middle East respiratory syndrome. Proc Natl Acad Sci U S A 111(13):4970-4975

8. Sarzi-Puttini P, Giorgi V, Sirotti S et al (2020) COVID-19, cytokines and immunosuppression: what can we learn from severe acute respiratory syndrome? Clin Exp Rheumatol 38(2):337-342

9. Novi G, Mikulska M, Briano F, Toscanini F, Tazza F, Uccelli A, Inglese M (2020) COVID-19 in a MS patient treated with ocrelizumab: does immunosuppression have a protective role? Mult Scler Relat Disord 42:102120

Publisher's note Springer Nature remains neutral with regard to jurisdictional claims in published maps and institutional affiliations. 